\title{
Food insecurity and human development, predict colorectal cancer in Colombia; an ecological study
}

\section{Abstract}

Introduction: Food insecurity (FI) has been associated with the generation of chronic disease and as a barrier in the treatment of cancer. We established at an ecological level the relationship between FI and the incidence rate of colorectal cancer $\mathrm{x} 100.000$ inhabitants (CRACI).

Materials and Methods: An ecological study was carried out with information that measured the level of human, social and economic development in the territory of the 33 geodemographic units that make up Colombia. Using multiple linear regression with the CRACI as the dependent variable, the FI as the main explanatory and other variables of the context, we established the relationship between FI and the CRACI and predictive equations of the CRACI by sex. The goodness of fit in the models was the coefficient of determination $\left(\mathrm{R}^{2}\right)$ and the Bland and Almant method.

Results: The FI is related in an inverse manner, and the human development Index (HDI) is directly related to the CRACI. Two equations predict the CRACI with high precision; In men, [CRACI $=-6.38+-0.25 * \mathrm{FI}+35.5 * \mathrm{HDI}]$.

In Women, [CRACI $=6.47+-0.23 * \mathrm{FI}+19.1 * \mathrm{HDI}$ ].

The coefficient of determination $\mathrm{R}^{2}(\%)$ in men is 88 and in women 72 . The average difference between CRACI observed versus the estimated, was -0.00 in men ( $95 \%$ CI: -3.13 to 3.13$)$, and in women of -0.00 (95\% CI: -4.08 to 4.08$)$.

Discussion: We establish that in Colombia, FI is significantly and inversely associated with the CRACI and that it is possible to predict the CRACI at an ecological level with a high degree of precision using two variables that are calculated on a regular basis by Colombian state agencies-FI and the HDI.

FI has previously been identified as a risk factor in the development and treatment of patients with cancer since the associated economic incapacity reduces adherence to treatment and promotes a limited biological response, explained by a limited and poor-quality diet that exposes these patients to greater levels of infection and relapse.

A possible explanation for this is that as greater social and economic development is achieved in the territory, decreasing FI, the diet transitions toward greater consumption of proteins of animal origin, which in turn favours the development of mediators of chronic diseases, such as obesity, hypertension, and cancer. This change is part of the phenomena called nutritional transition and food transition.

The equations presented here constitute a cost-effective method for estimating CRACI in Colombia.

\section{Conflict of Interest}

There is no conflict of interest 